\title{
Single-trial FRPs: A Machine Learning Approach Towards the Study of the Neural Underpinning of Reading Disorders
}

\author{
Christoforos Christoforou, Timothy C. Papadopoulos, Maria Theodorou \\ Department of Computer Science, St. John's University, New York, NY, USA, \\ Department of Psychology, University of Cyprus, Nicosia, Cyprus \\ christoc@stjonhs.edu, papadopoulos.timothy@ucy.ac.cy, mtheodorou@,rkileaders.com
}

\begin{abstract}
Understanding the neural underpinning of reading disorders, such as dyslexia, is a fundamental question in developmental neuroscience. However, identifying and isolating informative neural components elicited during free-naming paradigms (i.e. unprompted and unconstrained naming tasks) has proven a challenging methodological task. These methodological barriers have hindered the study of the neural underpinnings of reading disorders. In this paper, we proposed a machine learning approach for detecting neural components during free-naming, overcoming much of the current methodological challenges. We propose a new neural-based metric to differentiate groups of children with dyslexia (DYS) and their chronological age controls (CAC) in a free-naming task. Our approach combines electroencephalography (EEG) and eye-tracking measures to generate single-trial fixation-related potentials (sFRPs) and formulate an optimization problem to extract naming-related neural components, informative of group differences. Our approach is validated on a real dataset involving children with dyslexia and CAC performing a Rapid-Automatized Naming (RAN) task. Our results demonstrate the validity of the proposed metric as an indicator of the neural-based markers of reading disorders. Importantly, our proposed framework provides a novel approach that can facilitate the study of neural correlates of reading disorders under paradigms current methods are unable to.
\end{abstract}

\section{Introduction}

Understanding the neural-underpinnings of reading disorders, such as dyslexia, is a fundamental problem in developmental neuroscience. Analysis of neurophysiological measurements (i.e., EEG) is often used to provide insights into the underlying cognitive processes involved during reading and identify differences between typically developing children and children with reading disorders (Breznitz, 2005, Loberg et al., 2019). To facilitate the analysis of neurophysiological signals during reading or naming, experi-

Copyright $(C) 2020$, by the authors. All rights reserved. mental paradigms are designed to simulate the reading or naming task by presenting sequences of stimuli (typically, single letters or words) one at a time at predefined time intervals. Current analysis methods require such prompted paradigms to trigger Event-Related Potentials (ERP) measurable stereotypical neural waveforms evoked in response to physical stimuli - which are interpreted as indices of cognitive processes (Bakos et al., 2020). However, such prompted naming tasks - to which the present study focuses - do not necessarily engage the same cognitive processes as conventional, free-naming (i.e., unprompted and unconstrained naming tasks). Identifying neurophysiological components elicited during free-naming tasks is likely to provide more relevant information about the underlying cognitive processes involved during reading, thus providing a better understanding of reading disorders' neural underpinnings (Bakos et al., 2020). However, existing methodological approaches for EEG analysis cannot isolate informative neuronal components during free-naming tasks because of the lack of experimenter-controlled time-locked events necessary to extract ERPs (Cohen et al., 2018). Thus, there is a need for novel computational methods to identify differential neuronal activity during free-naming tasks (i.e., in the absence of experimenter-controlled timelocked events).

Traditionally, ERPs analysis has been used to differentiate neuronal responses among groups and conditions (Breznitz, 2005). The analysis involves averaging neural responses to time-locked events in order to generate an average estimate of the event-related potential waveform in response to each event. Subsequently, comparing differences in the average waveform between groups or conditions provides insights into the potential underlying neuronal processes that affect different cognitive processes. ERPs have been used to study several psychological processes, such as attention, memory and perception, personality traits, and intelligence. Yet, ERP analysis requires experimental designs with time-locked events, which are 
not available in free-naming paradigms, and thus cannot be directly applied to these paradigms.

Several machine learning approaches have also been proposed to analyze EEG in the study of neurocognitive processes. Such methods aim to identify neural correlates of different cognitive functions and often find application in scenarios where traditional average ERP cannot capture the true nature of the underlying neural components or overcome the ERP analysis's intrinsic limitations. For example, multivariate, single-trail discriminant analysis has been proposed to capture neural representations of decision-making processes during perceptual categorization tasks (Philiastides \& Sajda, 2005). Multivariate single-trial correlation analysis has been proposed to isolate neural components that maximally correlate with continuous, behavioral observations. It has been applied towards the characterization of stimulus presentation modality effect (i.e., the differences in performance observed in memory rehabilitation programs due to the modality of the stimuli used) (Christoforou et al., 2013). Common Special Patterns (CSP) based single-trial analysis has been proposed for cognitive process disambiguation in the context of special cognition (Christoforou et al., 2018). Similar to the ERP analysis, such methods have been employed only in paradigms that provide experimenter-controlled events and do not generalize to the free-naming type of paradigms.

Therefore, major barriers in applying such data-driven methods towards free-naming paradigms are the lack of time-locked events relevant to reading and the intrinsic low signal-to-noise ratio of EEG signals. These barriers are observed because of eye movement during free-naming tasks and the inter-subject variability observed in neurophysiological experiments (Christoforou et al. 2010). In this paper, we propose a new framework that allows for extracting neural components during free-naming tasks. Our approach utilizes eye-tracking and EEG measurements simultaneously recorded during a free-naming task to isolate neuronal activity that differentiates between children with dyslexia and their chronological age controls. Our analysis serves as a feasibility study for using machine learning techniques and multi-modal integration to study reading disorders during free-naming tasks.

\section{Methods}

\section{Experimental Paradigm and Data Collection}

Data used to assess the feasibility of the proposed methodology were collected as part of a broader project ${ }^{1}$ aiming to identify the neural underpinnings of dyslexia in children. Here, we briefly introduce the specific paradigm and data

\footnotetext{
${ }^{1}$ Fella A. \& Papadopoulos, T. C. (2017). Reading ability: Cognitive and neurophysiological performance indicators. Center for Applied Neuroscience, University of Cyprus.
}

collection apparatus related to the scope of the paper. In particular, we focus on analyzing data collected during a computerized version RAN task involving four different conditions. A total of 60 children ( 30 with dyslexia and 30 chronological-age controls) participated in the study; all children were native Greek speakers. At each task of the paradigm, participants were shown letter-matrix organized in five rows and ten columns and were asked to name out loud each letter from left to right and from top to bottom as fast and accurately as possible. Four letter-matrix conditions were shown to each participant, varied in the degree of visual and phonological confusability, among letters. An example of a letter-matrix condition (phonological confusable) is shown in Figure 1.

\begin{tabular}{llllllllll|}
$\beta$ & $\theta$ & $\alpha$ & $\varepsilon$ & $u$ & $\alpha$ & $\theta$ & $\beta$ & $u$ & $\varepsilon$ \\
$u$ & $\beta$ & $\theta$ & $\alpha$ & $\varepsilon$ & $u$ & $\alpha$ & $\theta$ & $\beta$ & $\varepsilon$ \\
$u$ & $\varepsilon$ & $\alpha$ & $\beta$ & $\theta$ & $\alpha$ & $\varepsilon$ & $u$ & $\theta$ & $\beta$ \\
$\theta$ & $u$ & $\varepsilon$ & $\alpha$ & $\beta$ & $\theta$ & $\alpha$ & $\varepsilon$ & $u$ & $\beta$ \\
$\theta$ & $\beta$ & $\alpha$ & $u$ & $\varepsilon$ & $\alpha$ & $\beta$ & $\theta$ & $\varepsilon$ & $u$ \\
\hline
\end{tabular}

Figure 1 Example stimuli from the Rapid-Automatized Naming Paradigm; phonologically confusable stimuli; beta and theta are phonologically similar.

Simultaneous Eye-tracking and EEG measurements were collected for the session duration. Eye-gaze data were recorded using the Eyelink 1000 eye-tracking system (SR Research) at a $1000 \mathrm{~Hz}$ sampling rate. To improve eye-gaze measurement stability, participants held their head in a chinrest for the duration of the data collection session. A 13-point calibration session was run before the experiment begin. In addition to raw eye-gaze points, eye-motion events such as fixations and saccades were recorded using EyeLink Parser. EEG data were collecting using a BioSemi Active-two system (BioSemi, Amsterdam, Netherlands) at a sampling rate of $256 \mathrm{~Hz}$. A 64 -electrode cap following the $10 / 20$ system was used; the DC offset of all sensors was kept below $20 \mathrm{mV}$. A trigger channel was used to synchronize measurements from both modalities to record time markers indicating each trial's beginning and end.

\section{EEG and Eye-tracking data pre-processing}

Fixation points of each participant were loaded using the pyGaze Analyzer library in python. The trigger channel information was used to re-reference and synchronize the time- onset between the two modalities. For each participant and stimulus, the set of recorded fixations whose timestamp fell within the stimulus's presentation duration were extracted and labelled as such. Fixations whose (x,y) 
coordinates fell outside the visual region of the stimulus or whose duration exceeded 3 standard deviations of the mean duration were removed. At the end of the pre-processing, each participant $p$ and each stimulus $s$ was associated with the corresponding set of valid-fixations $F_{p, s}=$ $\left\{f_{1}, \ldots f_{n}\right\}$. Each fixation $f_{i}$ was represented by its $(\mathrm{x}, \mathrm{y})$ coordinates, its starting onset (relative to the stimulus synch onset), and a duration time, all measured in milliseconds.

All EEG pre-processing was performed using custom Python code and using the MNE library ${ }^{2}$. A $0.5 \mathrm{~Hz}$ highpass filter was used on the continuous EEG to remove DC drifts, and notch filters at $50 \mathrm{~Hz}$ and $100 \mathrm{~Hz}$ were used to minimize the power-line interference. EEG sub-segments, spanning 2 seconds before each stimulus onset and until 2 seconds after the stimulus offset, were generated. The baseline amplitude of each segment was removed, and channels were re-referenced to the average reference. The duration of each segment varied between each participant and each stimulus. Thus each participant was free to name the stimuli at their own pace. At the end of the basic EEG pre-processing, each participant and stimulus has an associate EEG segment, $E E G_{p, s} \in \mathbb{R}^{D \times T_{p, s}}$ representing the entire recording session of the segment of $\mathrm{D}$ channels and $T_{p, s}$ time samples.

\section{Generating single-trial Fixation Related Potentials}

As noted in the introduction, free-naming tasks, such as the RAN task used in this study, do not provide experimentercontrolled time-locked trials necessary to extract EventRelated Potentials. This makes the application of conventional single-trail analysis methods not directly applicable. To overcome this inherent constraint of the paradigm and enable the study of naming under realistic scenarios, we explored integrating eye-gaze measures and EEG measures to identify and detect differential neural components. Specifically, the fixation and EEG segments were used to generate a set of Fixation Related Potentials (FRPs), i.e. EEG responses following the onset of a fixation. In particular, for each EEG segment $E E G_{p, s}$ (i.e. the EEG recording of a participant $p$ during the stimuli presentation $s$ ), and the corresponding set of fixations $F_{p, s}$, a set of $\left|F_{p, s}\right|$ FRPs were generated by epoching the $E E G_{p, s}$ segment, between at $-200 \mathrm{~ms}$ to $500 \mathrm{~ms}$ of the onset time of each $f_{i} \in F_{p, s}$. Baseline amplitude was removed from each FRP. Moreover, each single-trial FRP was assigned a group label $y_{p, s}(t) \in\{0,1\}$ indicating whether the participant generating the trial originated from the DYS and CAC groups.
After epoching, the resulting dataset is a set of single-trial FRPs and corresponding group labels as follows:

$$
F R P s=\left\{X_{p}(s, t) \in \mathbb{R}^{D \times T}, y_{p}(s, t) \in\{0,1\}\right\}_{t=1}^{T_{p, s}} \forall p, s
$$

where $\mathrm{D}$ corresponds to the number of channels and $\mathrm{T}$ the time samples in each FRP.

\section{Inter-subject FRP Correlation Components}

Our objective is to identify neural components in the resulting FRPs modulated by the naming task and carry differential information about CAC and DYS. Our approach is motivated by the hypothesis that synchrony of neural responses between individuals while naming is predictive of reading difficulty conditions and thus carries information about the underlying cognitive conditions. Here we provide details of the proposed approach to isolate such components.

Consider the set of single-trial FRPs $\left\{X_{p, s}(t) \in\right.$ $\left.\mathbb{R}^{D \times T}\right\} \forall p, s$, as defined in the previous section; we seek to find special multivariate components $\boldsymbol{w} \in \mathbb{R}^{D}$ that maximizes the inter-subject Pearson correlation of the single-trial FRP projected on $\boldsymbol{w}$ for a set of conditions and all trials. Specifically, for a pair of participants $(\mathrm{i}, \mathrm{j})$, and a given spatial projection $\boldsymbol{w}$ the inter-subject Pearson Product Moment Correlation Coefficient between the projected components is given by:

$$
\frac{\frac{1}{|S| .|T|} \sum_{s \in S} \sum_{t \in T} \boldsymbol{w}^{T} X_{i}(s, t) X_{j}^{T}(s, t) \boldsymbol{w}}{\left.\prod_{k \in\{i, j\}}\left(\frac{1}{|S||T|} \sum_{s \in S} \boldsymbol{w}^{T} X_{k}(s, t) X_{k}^{T}(s, t) \boldsymbol{w}\right)\right)^{1 / 2}}
$$

where $S=\{1,2,3,4\}$ denotes the set of the four stimulus types, $T=\{1, \ldots, N\}$ denotes in indexes of the set of singletrials within each stimulus, $|$.$| denotes the cardinality of a$ set. The above equation can be re-written in terms of the covariance matrix of the single-trial observation as follows:

$$
\frac{\boldsymbol{w}^{T} R_{i j} \boldsymbol{w}}{\left(\boldsymbol{w}^{T} R_{i i} \boldsymbol{w}\right)^{1 / 2}\left(\boldsymbol{w}^{T} R_{j j} \boldsymbol{w}\right)^{1 / 2}}
$$

where

$$
R_{i j}=\frac{1}{|S| .|T|} \sum_{s \in S} \sum_{t \in T} X_{i}(s, t) X_{j}^{T}(s, t)
$$

With that, we seek to find the optimal spatial projector vector $\boldsymbol{w}$ that maximizes the average Person Product Moment Correlation Coefficient across all subject pairs. Formally, the optimization problem seeks to find $\boldsymbol{w}$ maximize:

\footnotetext{
${ }^{2}$ https://mne.tools/stable/index.html
} 
$\arg _{w} \max \frac{1}{N} \sum_{i \in P} \sum_{j \in P} \delta_{i j} \frac{\boldsymbol{w}^{T} R_{i j} \boldsymbol{w}}{\left(\boldsymbol{w}^{T} R_{i i} \boldsymbol{w}\right)^{-1 / 2}\left(\boldsymbol{w}^{T} R_{j j} \boldsymbol{w}\right)^{-1 / 2}}$

where $P$ is the set of participants, $N=P(P-1)$ and $\delta_{i, j}=$ 1 if $i \neq \mathrm{j}, 0$ otherwise. Under the assumption that $\boldsymbol{w}^{\boldsymbol{T}} R_{i i} \boldsymbol{w} \approx \boldsymbol{w}^{T} R_{i i} \boldsymbol{w} \forall(i, j)$ the optimal $\boldsymbol{w}$ is given by the solution to the following generalized eigenvalue problem (see appendix for derivation):

$$
R^{(b)} \boldsymbol{w}=\lambda R^{(w)} \boldsymbol{w}
$$

where

$$
\begin{gathered}
R^{(b)}=\frac{1}{N} \sum_{i \in P} \sum_{j \in P} \delta_{i j} R_{i j} \\
R^{(w)}=\frac{1}{P} \sum_{i \in P} R_{i i}
\end{gathered}
$$

The solution to the generalized eigenvalue problem above comprise the $\mathrm{K}$ eigenvectors $\left\{\boldsymbol{w}_{k}\right\}_{k=1}^{K}$ of the matrix $\left(R^{(w)}\right)^{-1} R^{(b)}$ and the corresponding eigenvalues $\lambda_{k}=$ $\frac{\boldsymbol{w}_{\boldsymbol{k}}^{T} R^{(b)} \boldsymbol{w}_{\boldsymbol{k}}}{\boldsymbol{w}_{\boldsymbol{k}}^{T} R^{(w)} \boldsymbol{w}_{\boldsymbol{k}}}$. Each eigenvector $\boldsymbol{w}_{k}$ represents a componentprojection that captures the largest correlations in neural activity between all participants, while its corresponding eigenvalue $\lambda_{k}$ denotes the strength of that correlation. Components associated with high eigenvalues correspond to neural responses similar across subjects during the task at hand. Thus, they likely represent neural activity which is modulated by the underlying cognitive states during naming. We refer to these components as naming-related cognitive-congruency components.

To determine (quantify) how similar each subject is to the others experiencing the same stimulus, we calculated a per-subject naming-congruency, which is computed as:

$$
\text { per_subject_ } \lambda_{p, k}=\frac{\boldsymbol{w}_{\boldsymbol{k}}^{T} R^{(b), p} \boldsymbol{w}_{\boldsymbol{k}}}{\boldsymbol{w}_{\boldsymbol{k}}^{T} R^{(w), p} \boldsymbol{w}_{\boldsymbol{k}}}
$$

where

$$
\begin{aligned}
& R^{(b), p}=\frac{1}{N-1} \sum_{i \in P} \delta_{i p}\left(R_{i p}+R_{p i}\right) \\
& R^{(w), p}=\frac{1}{N-1} \sum_{i \in P} \delta_{i p}\left(R_{i i}+R_{p p}\right)
\end{aligned}
$$

note that $\delta_{i, j}=1$ if $i \neq \mathrm{j}, 0$ otherwise, where the set of $\left\{\boldsymbol{w}_{k}\right\}_{k=1}^{K}$ corresponds to the eigenvectors of equation (1).

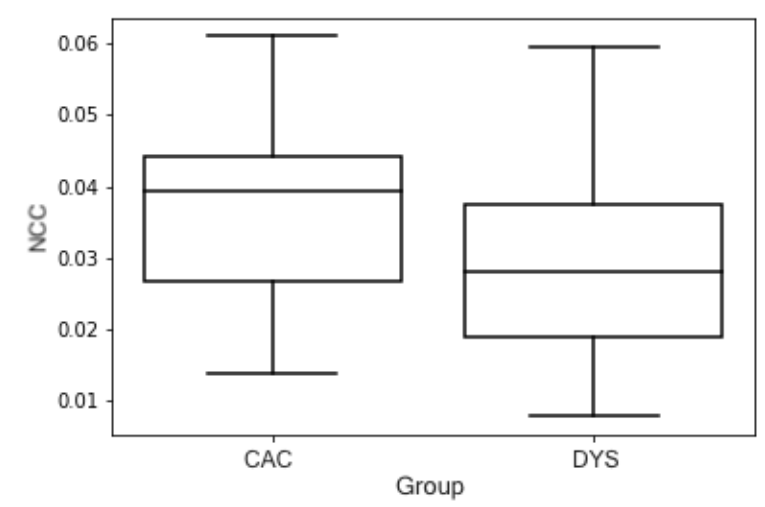

Figure 2:Box plot of the average NCC metric for each group

Naming speed-related Cognitive Congruency Metric

We consider the sum over the per-subject namingcongruency components as a metric of the overall neural activity relevant to the naming task. In particular, we define the Naming-related Cognitive Congruency (NCC) as:

$$
N C C(p)=\sum_{k \in K^{\prime}} \text { per_subject_ } \lambda_{p, k}
$$

for a selected subset of $K^{\prime}$ cognitive-congruency components. The resulting $\mathrm{NCC}(\mathrm{p})$ score corresponds to a metric that captures the overall synchrony in neural activity during naming per subject (i.e., participant $p$ ). Thus, it could serve as a neural-related metric to naming speed.

\section{Spatial and temporal profile of naming-related cognitive congruency components.}

Given the solutions to the generalized eigenvalue problem, the temporal profile of each component is calculated as the product of each component $\boldsymbol{w}_{k}$ with each of the single-trial FPR, followed by taking $t$ grant-average FRP of the projected components. Moreover, the topographical profile (i.e., the forward model) of each component is calculated as:

$$
\boldsymbol{a}_{k}=\frac{R^{(w)} \boldsymbol{w}_{k}}{\boldsymbol{w}_{k}^{\mathrm{T}} R^{(w)} \boldsymbol{w}_{k}}
$$

The forward model captures the covariance between each component's activity as measured by each electrode.

\section{Statistical comparison between groups}


To compare differences between DYS and CAC groups to determine the discriminative power of the proposed naming-related cognitive congruency metric, we employ an ANOVA test.

\section{Results}

We sought to investigate whether the proposed namingrelated cognitive-congruency metric is predictive in differentiating between DYS and CAC groups during the RAN task. We consider group membership (DYS vs CAC) as an independent variable and the proposed naming speedrelated cognitive congruency metric as a dependent variable. A one-way ANOVA analysis shows significant group differences between DYS and CAC $F(56,1)=7.20, p<0.01$. The boxplot in Figure 2 indicates that the average NCC score is significantly higher for the CAC group than the DYS group.
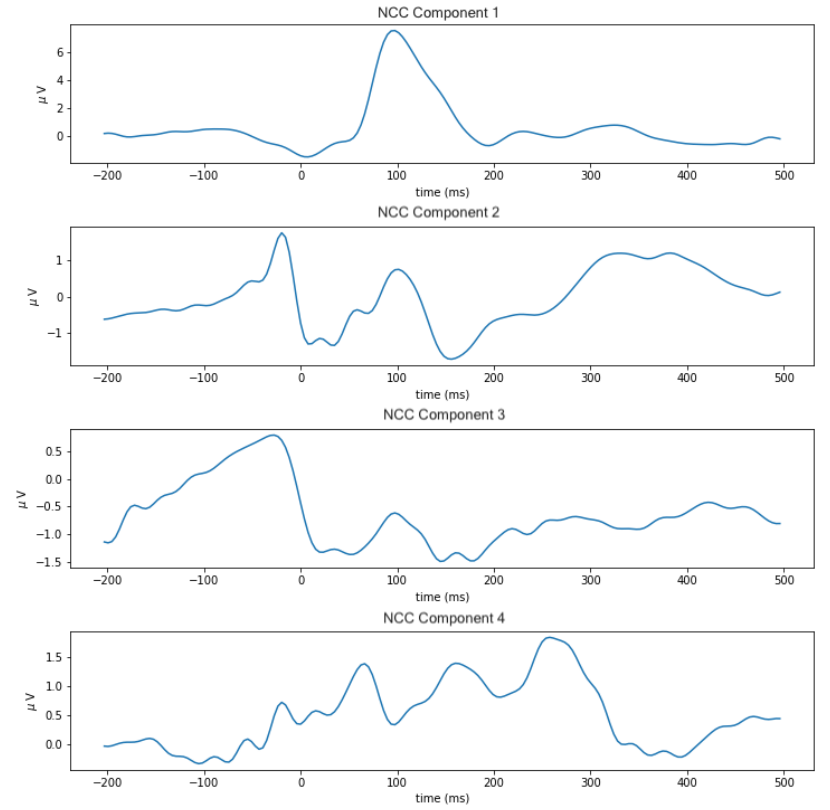

Figure3: Temporal profiles of the NCC components with the highest eigenvalue

Figure 3 shows the projected NCC component average across all FRPs of the four components with the largest eigenvalues in descending order (i.e., component 1 has the highest lambda). The topographic profile for the four components is illustrated in Figure 4.

\section{Discussion and Conclusion}

We proposed a novel computational approach to identify neural components elicited during an unconstrained RAN task that differentiates children with dyslexia and their chronological age controls under four different conditions. In particular, we formulated an optimization problem to extract spatial components from EEG measures that max-
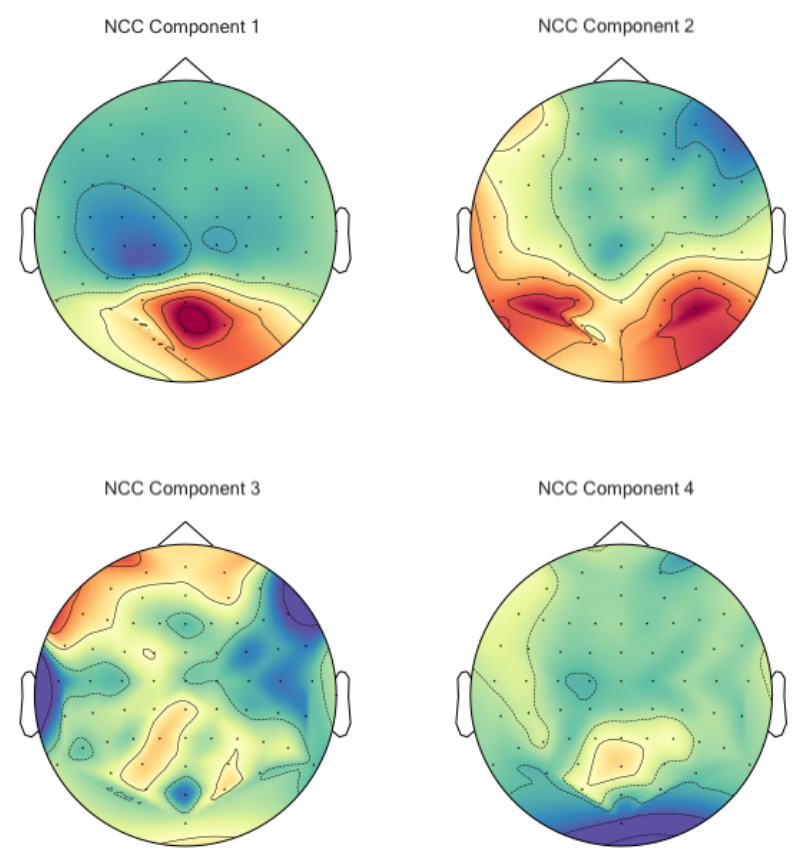

Figure 4: Forward model of the NCC components with the highest eigenvalue.

imize the correlation between fixation-related potentials during naming speed. Based on the resulting optimal components, we then defined the per-participant Namingrelated Cognitive Congruency metric (i.e., NCC), which served as an indicator of the degree to which participants engaged neural processes relevant to the naming task. Moreover, the resulting components are associated with corresponding temporal and spatial profiles, which allow us to investigate and interpret the spatiotemporal origins of the underlying neural activity. They also help study the neural underpinning of reading disorders under realistic naming paradigms (i.e. unprompted naming speed task). The proposed NCC metric is validated to carry predictive information between DYS and CAC groups in a real dataset.

This study's key finding is novel empirical evidence that the proposed NCC metric captures neural components that carry information that differentiates between DYS and CAC. Our results show that the resulting NCC differ significantly between the two groups, with the CAC group exhibiting stronger NCC scores than the DYS group. This result suggests that the neural responses during a naming speed task follow a stereotypical pattern more consistently for chronological age controls than for DYS, who deviate from the expected pattern. Our results also suggest that single-trial fixation-related potentials encode neural activity of the underlying naming-related cognitive processes that can be isolated using our proposed method.

The proposed method overcomes several methodological challenges allowing extracting neural components dur- 
ing unconstrained naming speed paradigms than the current state-of-the-art method. In particular, it eliminates the need for experimenter's controlled onsets by integrating fixation-related onset to define single-trials. Moreover, it mitigates inter-subject variability in single-trial analysis methods by maximizing the correlation of the resulting components across subjects.

Finally, our study's broader impact is that it provides a methodological approach to facilitate research on studying the neural underpinning of reading disorders during reading paradigms. By isolating neural components, our approach allows for identifying task-relevant neural activity under realistic naming tasks which are expected to be similar in reading paradigms (e.g., Georgiou, Parrila, Papadopoulos, \& Cui, 2013). In future work, we will explore the use of the NCC to characterize the reading components under different difficulty levels (i.e. congruent vs incongruent) and under different age groups.

\section{Reference}

Bakos, S.; Mehlhase, H.; Landerl, K.; Bartling, J.; Schulte-Körne G.; and Moll, K. 2020. Naming processes in reading and spelling disorders: An electrophysiological investigation, Clinical Neurophysiology, 131, 351-360.

Breznitz, Z. (2005). Brain activity during performance of naming tasks: Comparison between dyslexic and regular readers. Scientific Studies of Reading, 9, 17-42.

Christoforou, C.; Constantinidou, F.; Shoshilou, P.; and Simos, P. (2013). Single-trial linear correlation analysis: application to characterization of stimulus modality effects. Frontiers in Computational Neuroscience, 7, 15.

Christoforou, C.; Haralick, R.M.; Sajda, P.; and Parra, L. C. (2010). The bilinear brain: Towards subject-invariant analysis, In 2010 4th International Symposium on Communications, Control and Signal Processing (ISCCSP), pp. 1-6. IEEE, 2010.

Christoforou, C.; Hatzipanayioti, A.; and Avraamides, M. (2018). Perspective-taking vs mental rotation: CSP-based single-trial analysis for cognitive process disambiguation. In Wang, S., Yamamoto, V., Jianzhong S., Yang Y., Jones, E., Iasemidis, L., Mitchell, T., (Eds.) Proceedings of International Conference, Brain Informatics (pp. 109-199). Arlington, TX, USA.

Cohen, M.; Mahé, G.; Laganaro, M.; and Zesiger, P. 2018. Does the relation between rapid automatized naming and reading depend on age or on reading Level? A behavioral and ERP study, Frontiers in Human Neuroscience, 12, 73.

Georgiou, G. K.; Parrila, R. K.; Cui, Y.; and Papadopoulos, T. C. (2013). Why is rapid automatized naming related to reading? Journal of Experimental Child Psychology, 115, 218-225.

Loberg, O.; Hautala, J.; Hamalainen, J.A.; and Leppanen, P.H.T. 2019. Influence of reading skills and word length on fixationrelated brain activity in school-aged children during natural reading, Vision Research, 165, 109-122.

Philiastides, M.G.; and Sajda, P. 2005. Temporal characterization of the neural correlates of perceptual decision-making in the human brain. Cerebral Cortex 16, 509-518.

\section{Appendix}

In this section, we provide the derivation of the solution to the optimization problem in section 2. Recall we are trying to maximize the expression in equation (1) for the vector w. Under the assumption that $\boldsymbol{w}^{\boldsymbol{T}} R_{i i} \boldsymbol{w} \approx \boldsymbol{w}^{T} R_{i i} \boldsymbol{w} \forall(i, j)$, we define the covariance matrix equation 1 , with respect to the average covariance matrix $R^{(w)}=\frac{1}{P} \sum_{i \in P} R_{i i}$ as follows:

$$
g(w)=\frac{1}{N} \sum_{i, j \in P} \delta_{i j} \frac{\boldsymbol{w}^{T} R_{i j} \boldsymbol{w}}{\left(\boldsymbol{w}^{T} R^{(w)} \boldsymbol{w}\right)}
$$

Taking the derivative of $\mathrm{g}(\mathrm{w})$ for $\mathrm{w}$ and setting it to zero, we get the following

$$
\begin{aligned}
& \frac{\partial g(\boldsymbol{w})}{\partial \boldsymbol{w}^{T}} \\
& =\frac{1}{N} \sum_{i, j \in P} \delta_{i j} \frac{R_{i j} \boldsymbol{w}\left(\boldsymbol{w}^{T} R^{(w)} \boldsymbol{w}\right)-R^{(w)} \boldsymbol{w}\left(\boldsymbol{w}^{T} R_{i j} \boldsymbol{w}\right)}{\left(\boldsymbol{w}^{T} R^{(w)} \boldsymbol{w}\right)^{2}} \\
& =\frac{1}{\left(\boldsymbol{w}^{T} R^{(w)} \boldsymbol{w}\right)^{2}}\left(\frac{1}{N} \sum_{i, j \in P} \delta_{i j} R_{i j}\right) \boldsymbol{w}\left(\boldsymbol{w}^{T} R^{(w)} \boldsymbol{w}\right) \\
& -R^{(w)} \boldsymbol{w}\left(\boldsymbol{w}^{T}\left(\frac{1}{N} \sum_{i, j \in P} \delta_{i j} R_{i j}\right) \boldsymbol{w}\right)
\end{aligned}
$$

Setting the derivative to zero, we get:

$$
\begin{gathered}
R^{(b)} \boldsymbol{w}\left(\boldsymbol{w}^{T} R^{(w)} \boldsymbol{w}\right)-R^{(w)} \boldsymbol{w}\left(\boldsymbol{w}^{T} R^{(b)} \boldsymbol{w}\right)=0 \\
=>R^{(b)} \boldsymbol{w}\left(\boldsymbol{w}^{T} R^{(w)} \boldsymbol{w}\right)=R^{(w)} \boldsymbol{w}\left(\boldsymbol{w}^{T} R^{(b)} \boldsymbol{w}\right) \\
=>R^{(b)} \boldsymbol{w}=R^{(w)} \boldsymbol{w} \frac{\left(\boldsymbol{w}^{T} R^{(b)} \boldsymbol{w}\right)}{\left(\boldsymbol{w}^{T} R^{(w)} \boldsymbol{w}\right)} \\
=>R^{(b)} \boldsymbol{w}=\lambda R^{(w)} \boldsymbol{w}
\end{gathered}
$$

where we set $\lambda=\frac{\left(w^{T} R^{(b)} w\right)}{\left(w^{T} R^{(w)} w\right)}$ and $R^{(b)}=$ $\left(\frac{1}{N} \sum_{i, j \in P} \delta_{i j} R_{i j}\right)$. Thus, the optimal $\mathbf{w}$ is a solution to the generalized eigenvalue problem of equation (1) 